\title{
Oridonin enhances the anticancer activity of NVP-BEZ235 against neuroblastoma cells in vitro and in vivo through autophagy
}

\author{
LI-DI ZHANG ${ }^{1 *}$, ZHEN LIU $^{1 *}$, HUA LIU $^{2}$, DONG-MEI RAN ${ }^{3}$, JIA-HUI GUO ${ }^{1}$, \\ BIN JIANG ${ }^{1}$, YING-LI WU ${ }^{4}$ and FENG-HOU GAO ${ }^{1}$ \\ ${ }^{1}$ Institute of Oncology, Shanghai Ninth People's Hospital, Shanghai Jiao Tong University School of Medicine, \\ Shanghai 200011; ${ }^{2}$ Department of Gastroenterology, The Tenth Hospital Affiliated to Tongji University, \\ Shanghai 200072; ${ }^{3}$ Department of Pathology, The Sixth People's Hospital of Zhengzhou City, Zhengzhou, \\ Henan 450015; ${ }^{4}$ Hongqiao International Institute of Medicine, Shanghai Tongren Hospital/Faculty of \\ Basic Medicine, Chemical Biology Division of Shanghai Universities E-Institutes, \\ Key Laboratory of Cell Differentiation and Apoptosis of the Chinese Ministry of Education, \\ Shanghai Jiao Tong University School of Medicine, Shanghai 200025, P.R. China
}

Received February 27, 2016; Accepted April 9, 2016

DOI: $10.3892 /$ ijo.2016.3557

\begin{abstract}
The aberrant activation of PI3K/Akt/mTOR signaling pathway plays an important role in the oncogenesis, prognosis and chemotherapy resistance of neuroblastoma. However, NVP-BEZ235, a potent dual PI3K and mTOR inhibitor have not shown beneficial effects on neuroblastoma especially in terms of apoptosis induction as a single agent. We therefore attempted to explore an effective combination regimen to enhance the anticancer activity of NVP-BEZ235. Interestingly, we found that oridonin, a natural biologically active compound extracted from the Chinese medicinal herb Rabdosia rubescens, combined with NVP-BEZ235 mark-
\end{abstract}

Correspondence to: Professor Feng-Hou Gao, Institute of Oncology, Shanghai Ninth People's Hospital, Shanghai Jiao Tong University School of Medicine, 639 Zhi Zao Ju Road, Shanghai 200011, P.R. China E-mail: fenghougao@163.com

Professor Bin Jiang, Institute of Oncology, Shanghai 9th People's Hospital, School of Medicine, Shanghai Jiao Tong University, Shanghai 200011, P.R. China

E-mail: jiangbinwcr@sjtu.edu.cn

*Contributed equally

Abbreviations: NB, neuroblastoma; MRD, minimal residual disease; PI3K, phosphatidyl inositol 3-kinase; mTOR, the mammalian target of rapamycin; CQ, chloroquine; 3-mA, 3-methyladenine; shRNA, short hairpin RNA; AO, acridine orange; EB, ethidium bromide; TUNEL, TdT-mediated dUTP nick-end labeling; FITC, fluorescein isothiocyanate; PI, propidium iodide; CR, complete remission; VGPR, very good partial remission

Key words: NVP-BEZ235, oridonin, neuroblastoma, autophagy, apoptosis edly induced apoptosis of neuroblastoma cells. Notably, the synergistic activation of the apoptotic pathway was accompanied with enhanced autophagy as evidenced by significant decreased p62 expression as well as upregulated conversion of LC3-II. Suppression of the Beclin-1, a core component of the autophagy machinery, by means of shRNA resulted in diminished synergistic antitumor effect. Furthermore, the co-treatment with oridonin and NVP-BEZ235 was also much more effective than either agent alone in inhibiting the growth of neuroblastoma xenografts and in inducing tumor cells apoptosis. Taken together, our results suggest that the combination of NVP-BEZ235 and oridonin is a novel and potential strategy for neuroblastoma therapy.

\section{Introduction}

Neuroblastoma (NB) is a malignant paediatric tumor of the sympathetic nervous system that presents as a highly heterogeneous disease, ranging from spontaneous regression to high risk of fatality (1-3). Although the overall survival of neuroblastoma has increased greatly with the advances of diagnostic methods and therapeutic treatment over the recent years, the cure rate and life quality for high-risk neuroblastoma have only improved marginally when given aggressive conventional treatment $(4,5)$. Due to issues like early osseous and (or) bone marrow metastasis and minimal residual disease (MRD), the effect of surgical operation in advanced neuroblastoma patients is limited. Therefore, combination chemotherapy, especially the molecular targeted agent-based combination, remains the dominant strategy in the field of neuroblastoma treatment and research.

Numerous pieces of evidence show that activation of PI3K/ Akt/mTOR pathway correlates with poor prognosis and chemotherapy resistance in neuroblastoma (6-8). NVP-BEZ235 is a potent novel dual PI3K and mTOR kinase inhibitor which has shown great inhibitory efficacy on colorectal, breast, nonsmall cell lung carcinoma, renal cancer, and sarcoma (9-13). 
This compound as a single agent has also been investigated in neuroblastoma, where it was shown ineffective in MYCN-non amplified neuroblastoma, which led us to speculate that NVP-BEZ235 might be better suited as a part of combination therapy (14). Oridonin is an ent-kaurane diterpenoid extracted from the plant Rabdosia rubescens which has been used as antimicrobial, anti-inflammation and antitumor agent in traditional Chinese medicine for thousands of years $(15,16)$. As regards its antitumor activity, previous studies have demonstrated that oridonin as a supplement may potentiate the therapeutic effects of anticancer drugs gemcitabine and imatinib in the treatment of pancreatic cancer and $\mathrm{Ph}^{+}$acute lymphoblastic leukemia separately $(17,18)$. However, it is currently unknown whether oridonin can strengthen the effects of NVP-BEZ235 in neuroblastoma treatment.

In this study, we investigated the effect of combined treatment with NVP-BEZ235 and oridonin on the growth of human neuroblastoma cells in culture and in a preclinical mouse model. The data indicated that oridonin significantly enhanced the inhibitory effect of NVP-BEZ235 on neuroblastoma, leading to synergistic cell apoptosis and inhibition of NB xenograft tumor growth.

\section{Materials and methods}

Reagent. NVP-BEZ235 was purchased from Novartis (East Hanover, NJ,USA) and dissolved in dimethylsulfoxide (DMSO) to a stock concentration of $1 \mathrm{mmol} / \mathrm{l}$. Oridonin (purity $>98 \%$ ) was purchased from Chengdu Must Bio-Technology Co., Ltd. (Chengdu, Sichuan, China) and dissolved in DMSO to a stock concentration of $10 \mathrm{mmol} / \mathrm{l}$. The stock solutions were wrapped in foil and maintained at $-20^{\circ} \mathrm{C}$.

Cell lines and cell culture. Neuroblastoma cell lines (SHSY-5Y, SK-N-MC) were obtained from the Shanghai Institutes for Biological Sciences (Shanghai, China) and were cultured in monolayer culture in RPMI-1640 medium (Gibco-BRL, Long Island, NY, USA) supplemented with $10 \%$ fetal bovine serum (FBS) (Gibco-BRL) and $1 \%$ penicillin-streptomycin (Gibco-BRL), under standard culture conditions $\left(37^{\circ} \mathrm{C}\right.$ and $5 \% \mathrm{CO}_{2}$ ). Cells in the logarithmic phase of growth were used in all experiments.

Cell Counting Kit- 8 assay. The cell proliferation was assessed by Cell Counting Kit-8 reagent (CCK-8; Dojindo Molecular Technologies, Inc., Kumamoto, Japan). SHSY-5Y and SK-N-MC cells were seeded at a density of $5 \times 10^{3}$ cells $/ 200 \mu \mathrm{l} /$ well in 96-well culture plates and allowed to settle overnight. Then the wells were treated with NVP-BEZ235 and/or oridonin for 12 or $24 \mathrm{~h}$. Finally, the cultured wells were treated with $20 \mu \mathrm{l} /$ well of CCK- 8 solution and incubated for $2 \mathrm{~h}$ and the optical density of wells was measured at $450 \mathrm{~nm}$ using a microplate reader (Bio-Rad, Hercules, CA, USA).

Flow cytometric analysis of cell cycle and apoptosis. Neuroblastoma cells were seeded onto 6-well plate at a density of $1 \times 10^{5}$ per well. After 24-h incubation, they were treated with NVP-BEZ235 and/or oridonin for $24 \mathrm{~h}$ and collected. For cell cycle analysis, cells were washed and fixed with ice-cold $75 \%(\mathrm{v} / \mathrm{v})$ ethanol at $-20^{\circ} \mathrm{C}$ for $2 \mathrm{~h}$, then after washing twice with PBS, the cells were suspended with $500 \mu 1$ PI solution (PI $50 \mu \mathrm{g} / \mathrm{ml}$ and RNase A $100 \mu \mathrm{g} / \mathrm{ml}$ ). The samples were analyzed by FACScan flow cytometer (BD, Franklin Lakes, NJ, USA). FITC Annexin V Apoptosis Detection Kit I (BD Pharmingen, San Jose, CA, USA) was used to quantify drug-induced cell apoptosis following the manufacturer's guidelines.

Acridine orangelethidium bromide (AO/EB) fluorescence. Neuroblastoma cells were cultivated in a 24-well plate exposed to NVP-BEZ235 and/or oridonin for $24 \mathrm{~h}$ in a humidified incubator $\left(37^{\circ} \mathrm{C}, 5 \% \mathrm{CO}_{2}\right)$. After removing the culture solution and washing with PBS, the cells were stained with $200 \mu$ l mixture of $(100 \mu \mathrm{g} / \mathrm{ml})$ acridine orange (AO) and $(100 \mu \mathrm{g} / \mathrm{ml})$ ethidium bromide (EB) (Sigma, St. Louis, MO, USA) with the 1:1 AO to $\mathrm{EB}$, and then incubated the plate for $3 \mathrm{~min}$ in the incubator. The cells were visualized by a fluorescence microscope (Nikon Eclipse Ti, Japan).

Lentiviral transduction. shRNA pLKO.1 lentiviral constructs were obtained from Hanbio (Shanghai, China). Sequences are as follows: Beclin-1 \#1, 5'-CCC GTG GAA TGG AAT GAG ATT-3'; Beclin-1 \#2, 5'-GCT TGG GTG TCC TCA CAA TTT-3'. Scrambled, 5'-GTG GAC TCT TGA AAG TAC TAT-3'. The pLKO.1 vectors and package plasmids (pLP1gag/pol, pLP2-Rev, pLP-VSVG) (Life Technologies, Carlsbad, CA, USA) were co-transfected into packaging 293T cells using Lipofectamine-3000 (Life Technologies). After $48 \mathrm{~h}$, the viral supernatants were collected and used to infect SH-SY5Y cells. Stable clones expressing the shRNAs were obtained via $5 \mu \mathrm{g} / \mathrm{ml}$ puromycin dihydrochloride (Santa Cruz Biotech Inc.; sc-205821) selection.

Western blot analysis. Cells were lysed with SDS lysis buffer containing phosphatase and protease inhibitor cocktail (Roche, Mannheim, Germany) and the protein concentration was assayed with Bio-Rad Protein Assay kit (Bio-Rad). Cell lysates with equal protein content were separated by SDS-polyacrylamide gel electrophoresis and transferred to PVDF membranes (Bio-Rad). After 3 washes, the membranes were blocked with $5 \%$ non-fat milk in Tris-buffered saline containing $0.1 \%$ Tween-20 (TBST, $\mathrm{pH} 7.4$ ) for $1 \mathrm{~h}$ at room temperature, followed by incubation overnight at $4^{\circ} \mathrm{C}$ with primary antibodies to cleaved caspase-3, cleaved PARP, cyclin D1, cyclin E1, p62, LC3B and Beclin-1 (all from Cell Signaling Technology, MA, USA). After washing 3 times, the membranes were incubated for $1 \mathrm{~h}$ at room temperature with species-specific HRP-conjugated secondary antibodies (Cell Signaling Technology). Immunoreactive bands were visualized using Immobilon Western chemiluminescent HRP substrate (Millipore Co., Billerica, MA, USA). Each experiment was performed at least 3 times independently. As a loading control, the GAPDH contents in the samples were also immunoblotted using GAPDH horseradish peroxidase-conjugated antibody (Cell Signaling Technology).

Neuroblastoma xenografts and treatments. Animal experiments were approved by the Institutional Animal Care and Use Committee (IACUC) of Shanghai Jiao Tong University. Five- to six-week old female athymic (nu/nu) mice were 
A

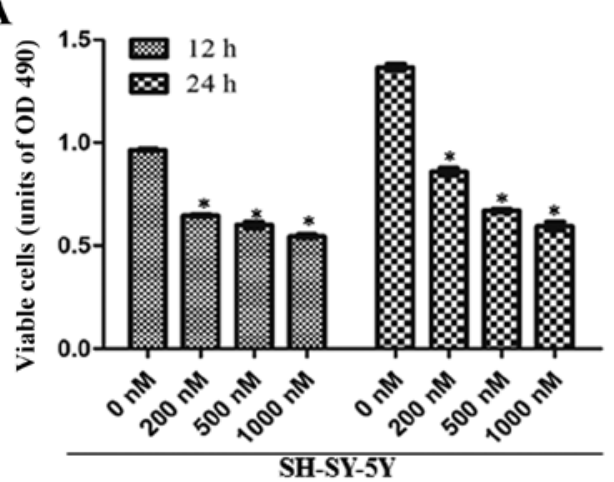

C

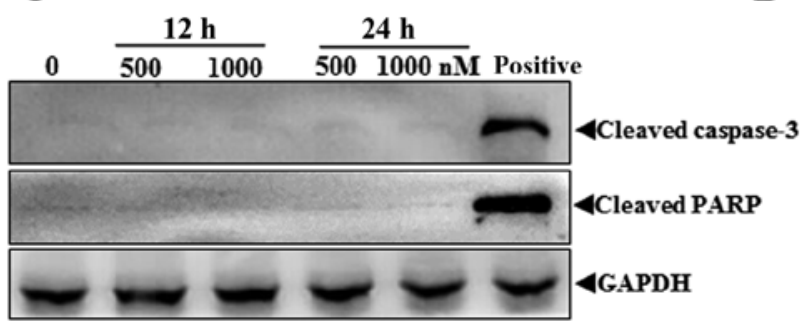

D
B
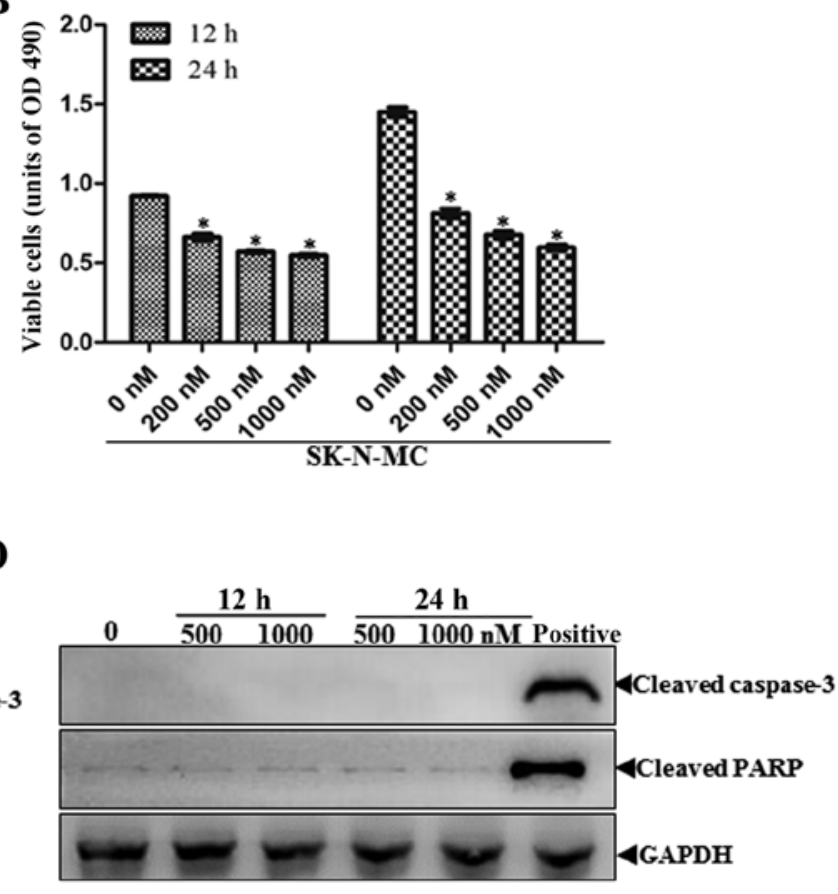

Figure 1. NVP-BEZ235 inhibits proliferation but does not induce apoptosis in neuroblastoma cell lines. (A and B) SHSY-5Y and SK-N-MC cells were treated with NVP-BEZ235 (200, 500 and 1,000 nM) for 12 or $24 \mathrm{~h}$. Cell viability was determined by CCK-8 assay. (C and D) Western blot analysis for the expression of cleaved caspase-3, cleaved PARP in SHSY-5Y and SK-N-MC cells treated with 500 and 1,000 nM NVP-BEZ235 for 12 and 24 h.

housed under pathogen-free conditions in micro-isolator cages with laboratory chow and water ad libitum. SHSY-5Y cells at $5 \times 10^{6}$ suspended in $100 \mu \mathrm{l}$ PBS were injected s.c. into the flank region of nude mice. When tumors were measurable and reached an average volume of $100 \mathrm{~mm}^{3}$, the mice were randomized into four groups for the following treatments: vehicle control, NVP-BEZ235 (20 mg/kg/day, i.p.), oridonin (10 mg/ $\mathrm{kg}$ /day; i.p.), and their combination. Tumor volumes were measured using caliper measurements once every 5 days and calculated with the formula $V=\pi$ (length $\mathrm{x}$ width $\mathrm{x}$ height) $/ 6$. Mice were sacrificed after 4 weeks of interventions and tumor tissues were excised and fixed in $10 \%$ buffered formalin and embedded in paraffin.

Histopathology and immunohistochemistry. Paraffinembedded blocks of all tumor samples were cut at $4 \mu \mathrm{m}$ and each sample was stained with hematoxylin and eosin (H\&E) for histologic analysis. For immunohistochemical detection of $\mathrm{Ki}-67$, sections were deparaffinized in xylene and ethanol and pretreated with a citrate buffer solution $(0.01 \mathrm{~mol} / \mathrm{l}$ citric acid and $0.01 \mathrm{~mol} / 1$ sodium citrate, $\mathrm{pH} 6.0$ ) in a microwave oven at $750 \mathrm{~W}$ for two cycles of $10 \mathrm{~min}$ each. Endogenous peroxidase activity was blocked by treatment with $3 \%$ hydrogen peroxide in methanol for $10 \mathrm{~min}$ at room temperature. After a blocking step with normal goat serum (Vector, Burlingame, CA, USA), the anti-Ki-67 antibodies (Cell Signaling Technology) were applied overnight in a moist chamber at $4^{\circ} \mathrm{C}$. The following day, tissue sections were incubated with a secondary biotinylated anti-rabbit antibody and with an avidin-biotin-peroxidase complex (Vector). The final reaction product was revealed by exposure to $0.03 \%$ diaminobenzidine (Sigma) and nuclei were counterstained with hematoxylin. Appropriate negative controls for the immunostaining were prepared by omitting the primary antibody step and substituting it with nonimmune rabbit serum. All samples were analyzed by scoring staining intensity.

TUNEL assay. To observe the degree of apoptosis, TUNEL assay was performed as per the manufacturer's protocol (In Situ Cell Death Detection kit, POD, Roche). All samples were analyzed by scoring staining intensity.

Statistical analyses. Each sample was analyzed in triplicate, and experiments were repeated three times. In all figures, error bars are standard deviations. Statistical analyses were performed by Microsoft Office Excel 2003 (Microsoft, Albuquerque, NM, USA) and Statisca ver. 10 (StatSoft, Tulsa, OK, USA). Differences between mean values were evaluated by the unpaired t-test. Differences were considered statistically significant at $\mathrm{P}<0.05$.

\section{Results}

NVP-BEZ235 inhibits proliferation but does not induce apoptosis in NB. To determine the effect of the dual kinase inhibitor NVP-BEZ235 on the proliferation of human neuroblastoma cells, SHSY-5Y and SK-N-MC were treated with varying concentrations of NVP-BEZ235 (0, 200, 500 and 1,000 nM) for 12 and $24 \mathrm{~h}$ and then cell viability was measured by CCK-8 assay. As shown in Fig. 1A and B, NVP-BEZ235 causes a timeand dose-dependent inhibition of cell proliferation in both SHSY-5Y and SK-N-MC cells. Based on the above results, we further investigated whether NVP-BEZ235-induced proliferation inhibition is associated with apoptosis. We detected the 


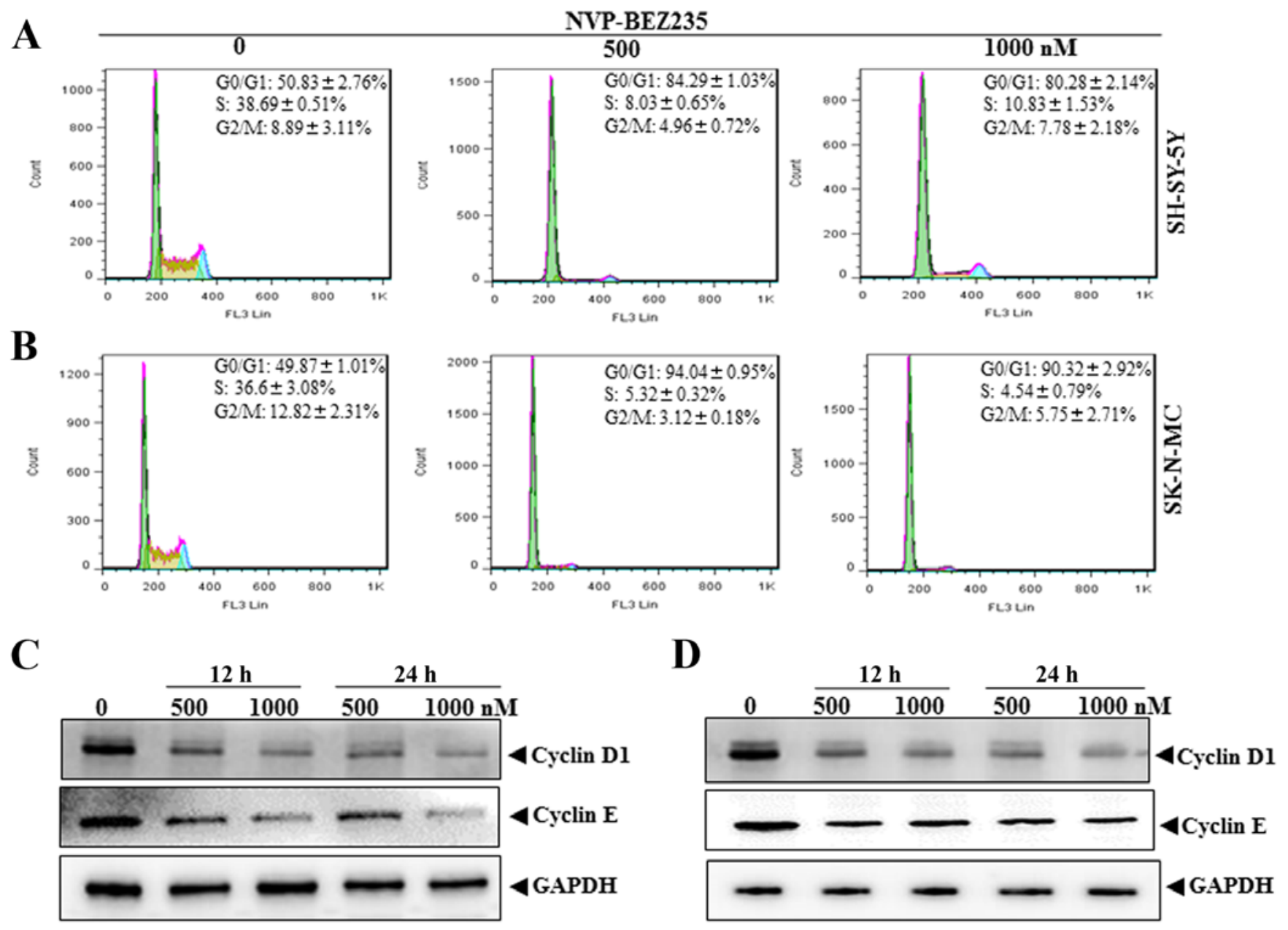

Figure 2. G1 phase blockade induced by NVP-BEZ235 treatments. (A and B) SHSY-5Y and SK-N-MC cells were treated with NVP-BEZ235 (500 and $1,000 \mathrm{nM}$ ) for $24 \mathrm{~h}$. Cellular DNA content was determined by staining with a hypotonic PI solution. (C and D) Western blot analysis of cyclins in SHSY-5Y and SK-N-MC cells treated with NVP-BEZ235 (500 and 1,000 nM) for 12 or $24 \mathrm{~h}$.

apoptotic relative proteins caspase- 3 and PARP cleavage in NVP-BEZ235 treated neuroblastoma cells. In contrast with the antiproliferative effects of NVP-BEZ235, it exerted little toxic effect, as indicated by the absence of cleaved caspase-3 and PARP in both treated cell lines (Fig. 1C and D).

NVP-BEZ235 induces G0/G1 cell cycle arrest in NB. As apoptosis could not have accounted for the potent inhibitory effect of NVP-BEZ235 on NB cell growth, we next analyzed the effect of NVP-BEZ235 on cell cycle progression in neuroblastoma cells by flow cytometry using PI staining of DNA content. Exposed to 500 and 1,000 nM NVP-BEZ235 for $24 \mathrm{~h}$, there was a significant increase in the percentage of SHSY-5Y cells in the G0/G1 phase $(84.29 \pm 1.03 \% 500 \mathrm{nM}, 80.28 \pm 2.14 \%$ $1,000 \mathrm{nM})$ compared with the control group $(50.83 \pm 2.76 \%)$ (Fig. 2A). The significant increase of G0/G1 phase cells was also detected in SK-N-MC cells (Fig. 2B). To further confirm NVP-BEZ235-induced G1 cell cycle arrest in neuroblastoma cells, we examined the expression of endogenous cyclins 12 and $24 \mathrm{~h}$ after NVP-BEZ235 treatment at 500 and $1,000 \mathrm{nM}$ in SHSY-5Y and SK-N-MC cells. Cyclin D1 and cyclin E1 are well-known G1-phase cyclins, governing the $\mathrm{G} 1 \rightarrow \mathrm{S}$ phase progression and their inhibition results in $\mathrm{G} 1$ phase arrest. As shown in Fig. 2C and D, NVP-BEZ235 treatment caused a remarkable reduction in cyclin D1 and cyclin E1 in both cell lines. Based on the above results, we concluded that the dual PI3K/mTOR inhibitor NVP-BEZ235 inhibits NB cell proliferation through the induction of cell cycle arrest but not apoptosis.

Co-treatment with NVPBEZ235 and oridonin induces enhanced antiproliferation and autophagy in NB. It is reported that enhanced autophagy induced by combination treatment can lead to programmed cell death and autophagic cell death has been proven to be effective in cases of solid tumors $(19,20)$. Given that NVP-BEZ235 alone can activate autophagy in neuroblastoma cells (Fig. 3A and B), we selected another autophagy related compound, oridonin, trying to combine with NVP-BEZ235 to improve its therapeutic efficacy. We first investigated the anti-proliferative effect of the combination treatment. The results showed that cell growth in both cell lines was markedly decreased following $24 \mathrm{~h}$ NVPBEZ235 and oridonin co-treatment when compared with either single agent alone (Fig. 3C and D). Then, we examined the autophagy activity of SHSY-5Y and SK-N-MC after the combination treatment through immunoblot analysis. As shown in Fig. 3E and F, there was substantially more LC3-II conversion after combination treatment compared with 
A

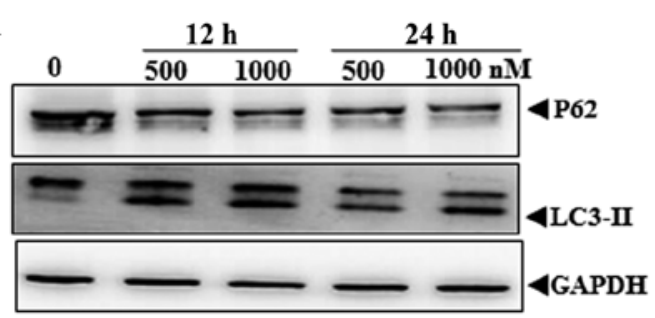

C

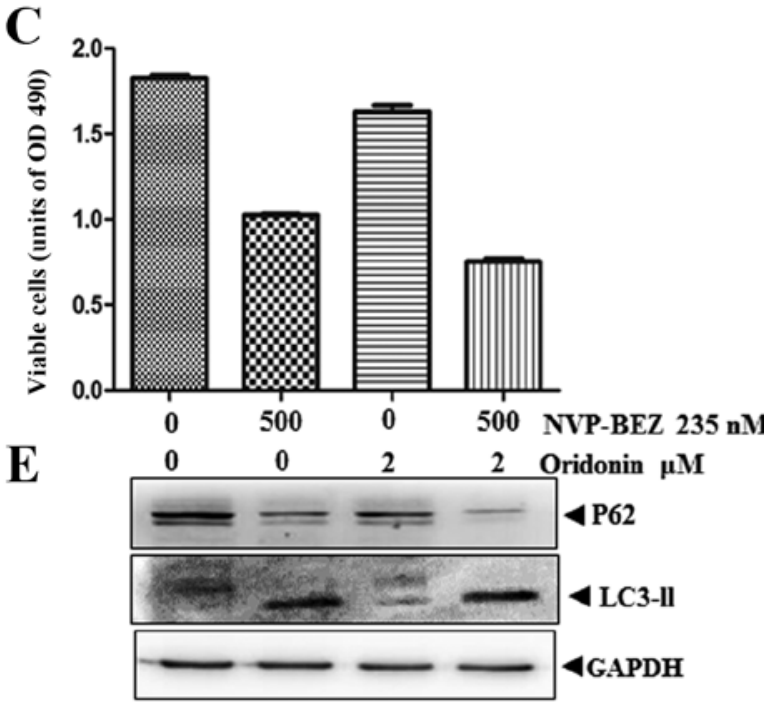

B

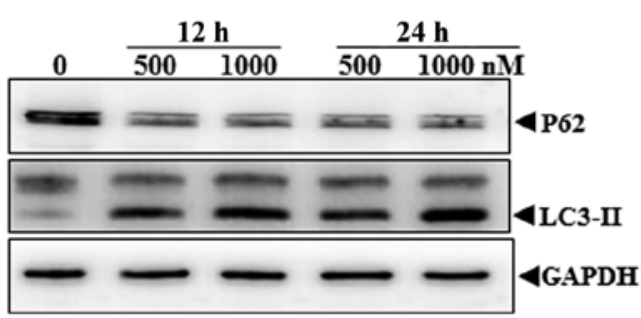

D

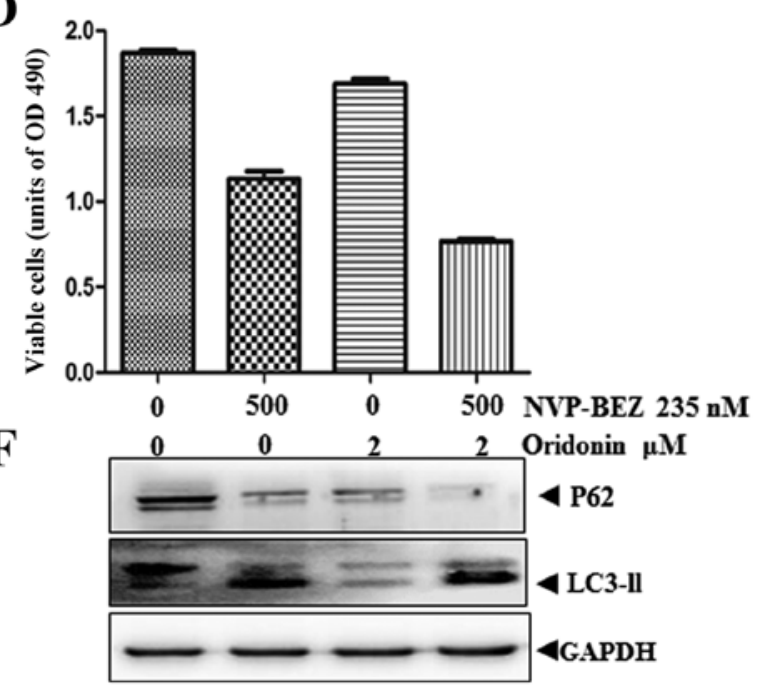

Figure 3. Effect of NVP-BEZ235 and/or oridonin on proliferation and autophagy in neuroblastoma cell lines. (A and B) Western blot analysis for the expression of p62 and LC3-I/II in SHSY-5Y and SK-N-MC cells treated with 500 and 1,000 nM NVP-BEZ235 for 12 or 24 h. (C and D) SHSY-5Y and SK-N-MC cells were treated with NVP-BEZ235 $(500 \mathrm{nM})$ and/or oridonin $(2 \mu \mathrm{M})$ for $24 \mathrm{~h}$. Cell viability was determined by CCK-8 assay. (E and F) Western blot analysis for the expression of p62 and LC3-I/II in SHSY-5Y and SK-N-MC cells treated with NVP-BEZ235 (500 nM) and/or oridonin (2 $\mu$ M) for $24 \mathrm{~h}$.

NVP-BEZ235 or oridonin treatment alone in both cell lines. In addition, co-treatment with NVP-BEZ235 and oridonin led to a significant decrease in the levels of p62 in the tested cells. This indicated that the combination of NVP-BEZ235 and oridonin can synergistically induce enhanced autophagy.

Co-treatment with NVPBEZ235 and oridonin induces apoptosis in $N B$. In order to examine whether the combination of NVP-BEZ235 and oridonin could synergistically induce apoptosis of $\mathrm{NB}$, the treated neuroblastoma cells were stained with $\mathrm{AO} / \mathrm{EB}$. AO was able to infiltrate into the viable cells, and the nuclei were stained a bright green color. For the integrity of the cell plasma membrane, EB was unable to infiltrate into the cells which remained alive or were at early stage of apoptosis, while the late apoptotic cells or dead cells had EB inside and the nuclei were stained a bright red color. Fig. 4A and $B$ show various morphologies of NB cells stained with AO/EB. After 500 nM NVP-BEZ235 treated, the number of viable cells in both cell lines was remarkably decreased in comparison with the control, while the cells cultivated with $2 \mu \mathrm{M}$ oridonin were similar to the control cells. More notably, the percentage of apoptotic cells was significantly increased in cells treated with the combination of NVP-BEZ235 and oridonin compared with either single agent alone. The results indicated the cell proliferation of NB was strongly inhibited by NVP-BEZ235, but it had very slight cell cytotoxicity compared to the combination groups which commit NBs to apoptosis. For further confirming the results, we examined the cleavage of caspase- 3 and PARP in the treated cells. As shown in Fig. 4C and D, when SHSY-5Y and SK-N-MC cells were treated with the combination of NVP-BEZ235 and oridonin for $24 \mathrm{~h}$, there was a significant increase of the cleaved form of caspase- 3 and PARP compared with either single agent alone, suggesting that apoptotic pathway was activated in response to the combination treatment.

Enhanced autophagy is essential for the induction of apoptosis by the co-treatment with NVP-BEZ235 and oridonin. To determine whether the enhanced autophagy is necessary for cell death induced by the combination treatment, we used shRNA to reduce the expression of Beclin-1 (Atg6), an integral component of the autophagic machinery, and examined whether its loss-of-function impacts on the combination-induced apoptosis. As expected, the expression level of Beclin-1 in SHSY-5Y cells was markedly reduced by shRNA treatment (Fig. 5A). To provide another level of confirmation, we performed flow cytometric analysis of Beclin-1 deficient cells stained with fluorescein isothiocyanate (FITC)-labeled Annexin V and propidium iodide (PI) to examine the population level of apoptotic response to the combination treatment. As shown in Fig. 5B, reduction of Beclin-1 protein decreased significantly the fraction of apoptotic cells in response to the co-treatment as quantified by the percentage of both early and late apoptotic cells. ShRNA-mediated knockdown of Beclin-1 led to a reduction of p62 degradation and LC3-II conversion and a significant rescue of cell death with the evidence of 
A

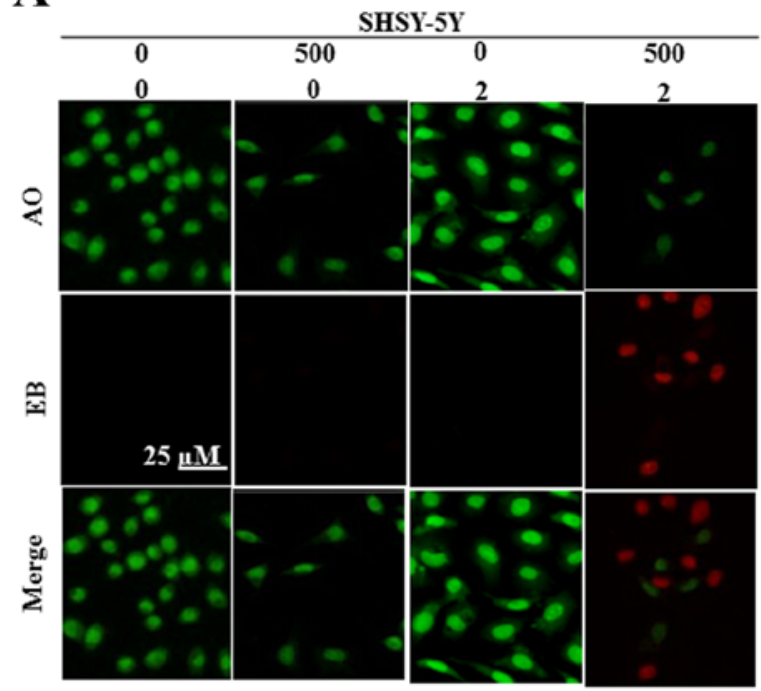

C

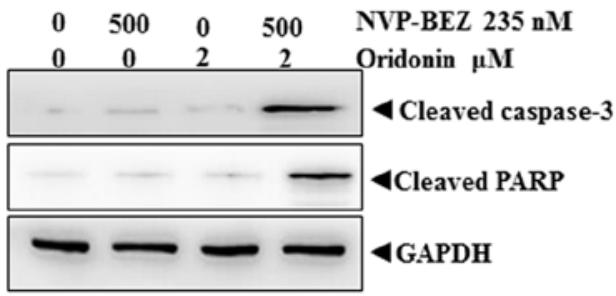

B

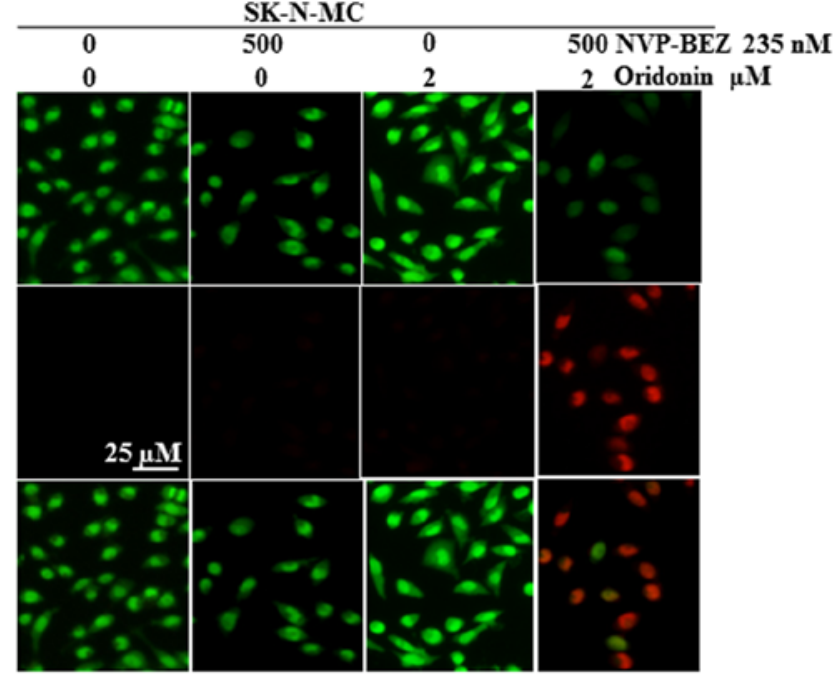

D

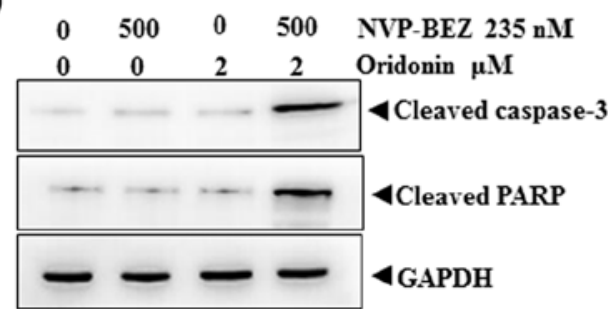

Figure 4. Effect of NVP-BEZ235 and/or oridonin on apoptosis in neuroblastoma cell lines. (A and B) Morphological changes in SHSY-5Y and SK-N-MC cells treated with NVP-BEZ235 $(500 \mathrm{nM})$ and/or oridonin $(2 \mu \mathrm{M})$ for $24 \mathrm{~h}$ followed by AO/EB staining (magnification, x100). (C and D) Western blot analysis for the expression of cleaved caspase-3 and cleaved PARP in SHSY-5Y and SK-N-MC cells treated with NVP-BEZ235 (500 nM) and/or oridonin (2 $\mu \mathrm{M})$ for $24 \mathrm{~h}$.

A

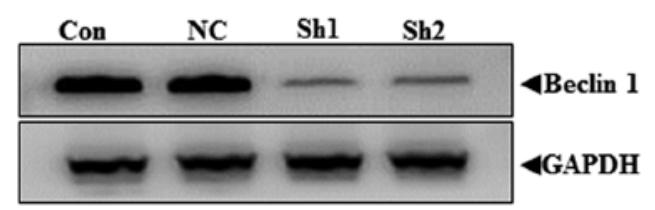

C

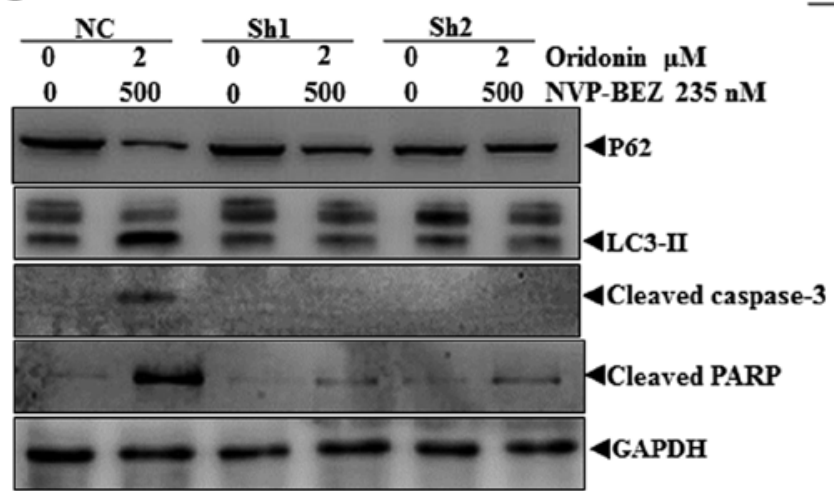

B

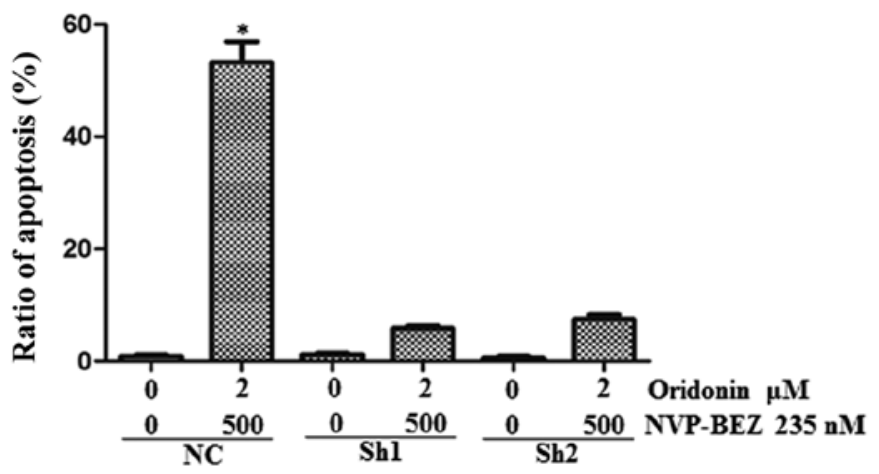

Figure 5. Enhanced autophagy is essential for the induction of apoptosis by the co-treatment with NVP-BEZ235 and oridonin. Western blot analysis for the expression of Beclin-1 in SHSY-5Y cells transfected with Beclin-1 shRNA. (B) Flow cytometric analysis of apoptosis with Annexin V-FITC/PI staining in Beclin-1 deficient cells exposed to the combination of NVP-BEZ235 $(500 \mathrm{nM})$ and oridonin $(2 \mu \mathrm{M})$ for $24 \mathrm{~h}$. (C) Western blot analysis for the expression of p62, LC3-I/II, cleaved caspase-3 and cleaved PARP in Beclin-1 deficient SHSY-5Y cells treated with the combination of NVP-BEZ235 (500 nM) and oridonin $(2 \mu \mathrm{M})$ for $24 \mathrm{~h}$. 
A

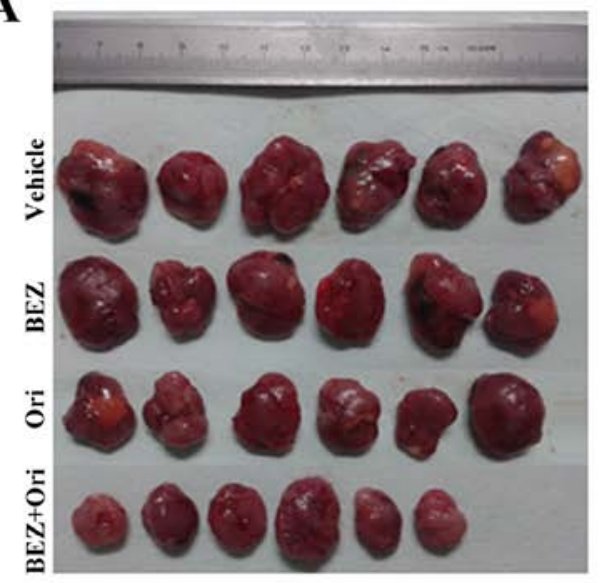

B

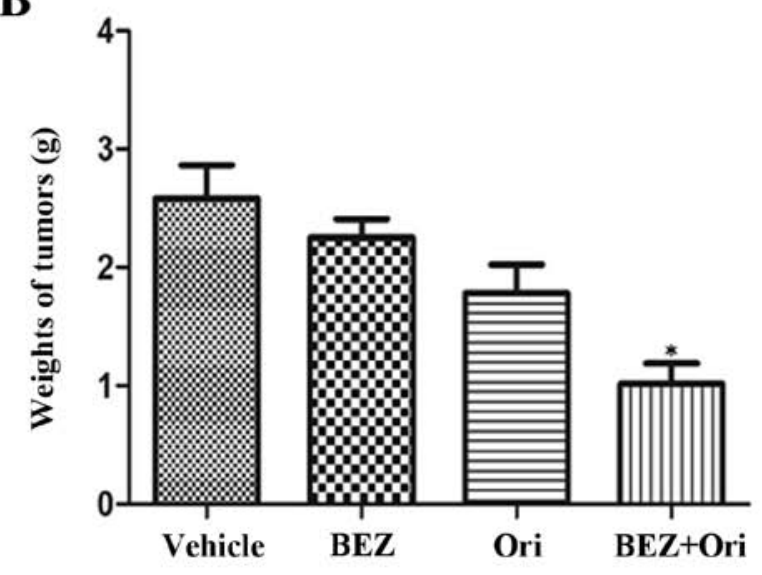

C

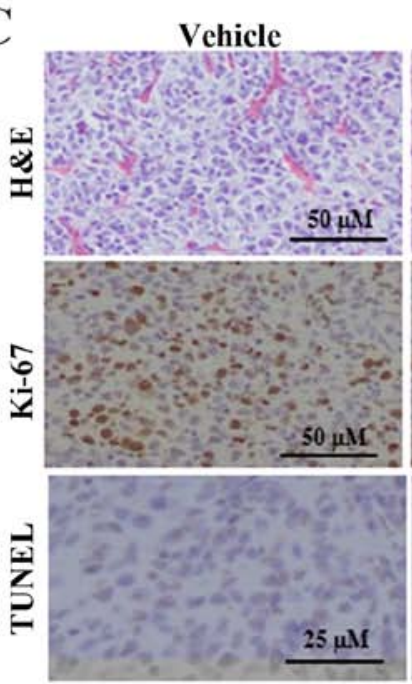

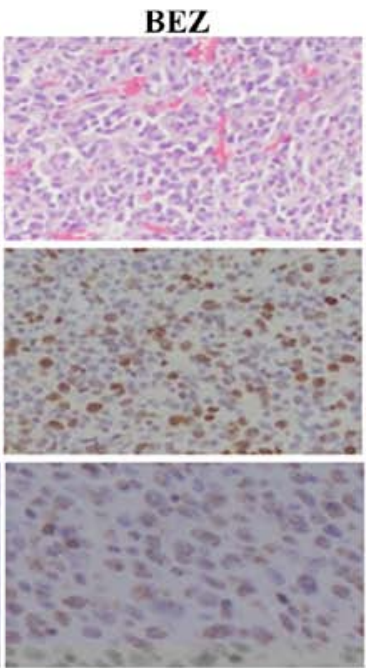

Ori

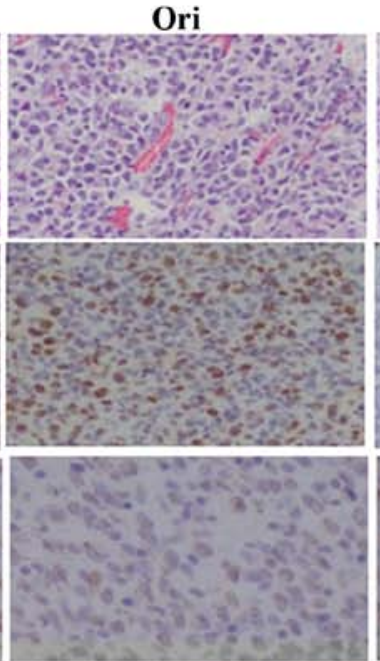

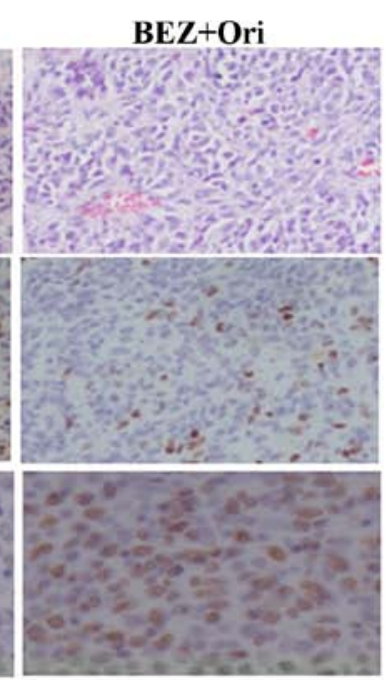

Figure 6. Antitumor activity of NVP-BEZ235 and/or oridonin in mouse xenograft models of neuroblastoma. Mice with SHSY-5Y xenografts were administered NVP-BEZ235 (20 mg/kg/day) and/or oridonin (10 mg/kg/day). After 4-week consecutive treatment, the mice were sacrificed. Tumors were resected from nude mice (A) and tumor weights (B) were measured at the end of observation. (C) Histopathological analysis of xenograft tumor tissues treated with NVP-BEZ235 and/or oridonin by H\&E, Ki-67 and TUNEL.

cleaved caspase- 3 and PARP absence in response to the combination treatment of NVP-BEZ235 and oridonin (Fig. 5C). These data strongly suggested that autophagy played a key role in the generation of antineoplastic effects of NVP-BEZ235 co-treatment with oridonin in neuroblastoma cells.

Co-treatment with NVP-BEZ235 and oridonin induces enhanced antitumor activity in NB xenografts. We next determined whether the synergism also exists in a preclinical NB xenograft mouse model. We initiated a tumor with subcutaneous injection of SHSY-5Y cells on the flank of nude mice. When tumor size reached $\sim 100 \mathrm{~mm}^{3}$, mice were intraperitoneally injected with vehicle, NVP-BEZ235 $(20 \mathrm{mg} / \mathrm{kg})$, oridonin $(10 \mathrm{mg} / \mathrm{kg})$, or their combination daily for 4 weeks and tumor sizes were measured every fifth day. As shown in Fig. 6A and B, the combination of NVP-BEZ235 and oridonin had a much more significant effect on suppression of tumor growth compared to any single drug. In addition, no significant changes in body weights or daily activities of tumor-bearing mice were observed after combination treatment, suggesting that the combination of these two drugs was well tolerated.
Furthermore, the proliferation and apoptosis index of tumor tissues were assessed by Ki-67 immunohistochemistry and TUNEL assay (Fig. 6C). Monotherapy with NVP-BEZ235 or oridonin only marginally decreased the proliferation of tumor tissues. While, consistently with the cytostatic effects induced by the combined treatment, proliferation of tumor tissues measured by Ki- 67 immunostaining was also markedly reduced in co-treated mice. Quantification of TUNEL staining did not reveal any significant change in apoptosis between vehicle control and NVP-BEZ235 or oridonin monotherapy group. However, there was significantly increased apoptosis of tumor tissues in combination treated mice as compared to vehicle control.

\section{Discussion}

The current chemotherapy strategies for the treatment of neuroblastoma have focused on intensifying and alternating combinations of cyclophosphamide, doxorubicin, vincristine, cisplatin, and etoposide $(21,22)$. Despite dose-intensive, multiagent induction chemotherapy, multicenter phase 3 trials 
suggested that the CR/VGPR rate is still around $50 \%(23,24)$. Given that conventional chemotherapeutics are nearing their maximum potential with regards to efficacy and patient tolerance, there is an urgent need to explore more effective and better-tolerated targeted therapy.

In this study, we observed that NVP-BEZ235 as a single agent can effectively block NB cell proliferation through inhibition of cell cycle progression but not activation of apoptosis, as evidenced by significant G1 phase arrest and the absence of caspase-3 cleavage. Consistent with this observation, Manara et al showed that NVP-BEZ235 induced sarcoma stasis, by arresting cells in G1 phase of the cell cycle, without remarkable effects on apoptosis (13). It was postulated that autophagy induced by NVP-BEZ235 was a pro-survival mechanism which rendered tumor cells capable of anti-apoptosis surviving in the kinase inhibitor (25-27).

Autophagy is an evolutionarily conserved mechanism by which cellular material is delivered to lysosomes for degradation and recycling $(28,29)$. Although previous reports suggest that the proper quantity of autophagy promotes malignant cell survival, there is emerging evidence that excessive autophagy modulates the cell death machinery in a positive way. Thus, it is conceivable that the induction of autophagy is cell contextdependent, and the extent of autophagy dictates the cellular outcome. For instance, there is evidence that autophagy promotes resistance of breast cancer cells to the monoclonal anti-HER2 antibody trastuzumab (30). Similarly, hypoxiainduced autophagy leads to chemoresistance of hepatocellular carcinoma cells $(31,32)$. On the other hand, there is evidence that autophagic cell death is critical for the generation of the effects of $\mathrm{As}_{2} \mathrm{O}_{3}$ on acute myelogenous leukemia cells (33) and successful induction of prolonged remissions of acute lymphoblastic leukemia by the combination of vincristine and RAD001 (34), implicating autophagy as a mechanism by which certain antineoplastic agents generate their antitumor activities. Hence, enhancement of NVP-BEZ235 induced autophagy may represent a new chemotherapy strategy for advanced neuroblastoma.

Therefore, oridonin, a natural compound that has been demonstrated to induce cell apoptosis and autophagy in several types of tumor cells, was selected to deal with NB cells together with NVP-BEZ235. The findings indicated that the combined treatment of the two drugs led to programmed death of NB cells in a synergistic manner, dramatically enhancing the antitumor efficacy on human NB cells in vitro and in vivo. Notably, the synergistic activation of the apoptotic pathway was accompanied by significant decreased p62 expression as well as upregulation of LC3-II. Knockdown of Beclin-1 which plays a key role in the initiation of autophagy pathway with shRNA led to a significant rescue of cell death with the evidence of cleaved caspase- 3 and PARP absence and decreased apoptosis rate from flow cytometry in response to the combination treatment of NVP-BEZ235 and oridonin. Such enhanced autophagy has also been reported by other studies to play an important role in synergic therapeutic effects induced by combination treatment. For instance, Tai et al showed that combined treatment with Rad001 and propachlor led to excessive autophagy, and resulted in cell death in prostate cancer cells (20). In regard to the mechanism of apoptosis promotion by excessive autophagy we speculated that apoptosis was promoted through autophagic degradation of negative regulators that controls the apoptosis machinery. Alternately, we supposed that autophagy induced by NVP-BEZ235 failed to reach the threshold to autophagic cell death, but it interrupted tumor intracellular homeostasis and enhanced the chemosensitivity of neuroblastoma cells to oridonin treatment. While the challenge remains to clearly understand the underlying mechanisms by which enhanced autophagy induced the combination of NVP-BEZ235 with oridonin to promote programmed cell death of NB.

These results showed that combination treatment of the two compounds synergize in the induction of NB cell death in vitro and in vivo, thus providing a novel strategy to develop combination therapies for advanced neuroblastoma patients that have failed the currently available therapies.

\section{Acknowledgements}

This study was supported in part by the National Natural Science Foundation of China (81172322 and 81302006), Shanghai Municipal Education Committee (13ZZ089), Science and Technology Committee of Shanghai (14401901500) and Science and Technology Committee of Baoshan District (12-E-2).

\section{References}

1. Berthold F, Boos J, Burdach S, Erttmann R, Henze G, Hermann J, Klingebiel T, Kremens B, Schilling FH, Schrappe M, et al: Myeloablative megatherapy with autologous stem-cell rescue versus oral maintenance chemotherapy as consolidation treatment in patients with high-risk neuroblastoma: A randomised controlled trial. Lancet Oncol 6: 649-658, 2005.

2. Park JR, Eggert A and Caron H: Neuroblastoma: Biology, prognosis, and treatment. Hematol Oncol Clin North Am 24: 65-86, 2010.

3. Westhoff MA, Faham N, Marx D, Nonnenmacher L, Jennewein C, Enzenmüller S, Gonzalez P, Fulda S and Debatin KM: Sequential dosing in chemosensitization: Targeting the PI3K/Akt/mTOR pathway in neuroblastoma. PLoS One 8: e83128, 2013.

4. Brodeur GM: Neuroblastoma: Biological insights into a clinical enigma. Nat Rev Cancer 3: 203-216, 2003.

5. Maris JM: Recent advances in neuroblastoma. N Engl J Med 362: 2202-2211, 2010

6. Opel D, Poremba C, Simon T, Debatin KM and Fulda S: Activation of Akt predicts poor outcome in neuroblastoma. Cancer Res 67: 735-745, 2007.

7. Fulda S: The PI3K/Akt/mTOR pathway as therapeutic target in neuroblastoma. Curr Cancer Drug Targets 9: 729-737, 2009.

8. Izycka-Swieszewska E, Drozynska E, Rzepko R, KobierskaGulida G, Grajkowska W, Perek D and Balcerska A: Analysis of $\mathrm{PI} 3 \mathrm{~K} / \mathrm{AKT} / \mathrm{mTOR}$ signalling pathway in high risk neuroblastic tumours. Pol J Pathol 61: 192-198, 2010.

9. Roper J, Richardson MP, Wang WV, Richard LG, Chen W, Coffee EM, Sinnamon MJ, Lee L, Chen PC, Bronson RT, et al: The dual PI3K/mTOR inhibitor NVP-BEZ235 induces tumor regression in a genetically engineered mouse model of PIK3CA wild-type colorectal cancer. PLoS One 6: e25132, 2011.

10. Chen X, Zhao M, Hao M, Sun X, Wang J, Mao Y, Zu L, Liu J, Shen Y, Wang J, et al: Dual inhibition of PI3K and mTOR mitigates compensatory AKT activation and improves tamoxifen response in breast cancer. Mol Cancer Res 11: 1269-1278, 2013.

11. Zito CR, Jilaveanu LB, Anagnostou V, Rimm D, Bepler G, Maira SM, Hackl W, Camp R, Kluger HM and Chao HH: Multilevel targeting of the phosphatidylinositol-3-kinase pathway in non-small cell lung cancer cells. PLoS One 7: e31331, 2012.

12. Ribback S, Cigliano A, Kroeger N, Pilo MG, Terracciano L, Burchardt M, Bannasch P, Calvisi DF and Dombrowski F: PI3K/ $\mathrm{AKT} / \mathrm{mTOR}$ pathway plays a major pathogenetic role in glycogen accumulation and tumor development in renal distal tubules of rats and men. Oncotarget 6: 13036-13048, 2015. 
13. Manara MC, Nicoletti G, Zambelli D, Ventura S, Guerzoni C, Landuzzi L, Lollini PL, Maira SM, García-Echeverría C, Mercuri M, et al: NVP-BEZ235 as a new therapeutic option for sarcomas. Clin Cancer Res 16: 530-540, 2010.

14. Chanthery YH, Gustafson WC, Itsara M, Persson A, Hackett CS, Grimmer M, Charron E, Yakovenko S, Kim G, Matthay KK, et al: Paracrine signaling through MYCN enhances tumor-vascular interactions in neuroblastoma. Sci Transl Med 4: 115ra3, 2012.

15. Li CY, Wang EQ, Cheng Y and Bao JK: Oridonin: An active diterpenoid targeting cell cycle arrest, apoptotic and autophagic pathways for cancer therapeutics. Int J Biochem Cell Biol 43: 701-704, 2011.

16. Liu Z, Ouyang L, Peng H and Zhang WZ: Oridonin: Targeting programmed cell death pathways as an anti-tumour agent. Cell Prolif 45: 499-507, 2012.

17. Liu DL, Bu HQ, Jin HM, Zhao JF, Li Y and Huang $\mathrm{H}$ : Enhancement of the effects of gemcitabine against pancreatic cancer by oridonin via the mitochondrial caspase-dependent signaling pathway. Mol Med Rep 10: 3027-3034, 2014.

18. Guo Y, Shan Q, Gong Y, Lin J, Yang X and Zhou R: Oridonin in combination with imatinib exerts synergetic anti-leukemia effect in $\mathrm{Ph}^{+}$acute lymphoblastic leukemia cells in vitro by inhibiting activation of LYN/mTOR signaling pathway. Cancer Biol Ther 13: 1244-1254, 2012.

19. Tang Y, Hamed HA, Cruickshanks N, Fisher PB, Grant S and Dent P: Obatoclax and lapatinib interact to induce toxic autophagy through NOXA. Mol Pharmacol 81: 527-540, 2012

20. Tai S, Sun Y, Liu N, Ding B, Hsia E, Bhuta S, Thor RK, Damoiseaux R, Liang C and Huang J: Combination of Rad001 (everolimus) and propachlor synergistically induces apoptosis through enhanced autophagy in prostate cancer cells. Mol Cancer Ther 11: 1320-1331, 2012.

21. Fong A and Park JR: High-risk neuroblastoma: A therapy in evolution. Pediatr Hematol Oncol 26: 539-548, 2009.

22. National Cancer Institute: Board PDQPTE: Neuroblastoma Treatment (PDQ(R)): Health Professional Version. In: PDQ Cancer Information Summaries National Cancer Institute (US), Bethesda (MD), 2002. http://www.cancer.gov/publications/pdq/ information-summaries/adult-treatment. Accessed, January 14, 2016.

23. Pearson AD, Pinkerton CR, Lewis IJ, Imeson J, Ellershaw C and Machin D; European Neuroblastoma Study Group; Children's Cancer and Leukaemia Group (CCLG formerly United Kingdom Children's Cancer Study Group): High-dose rapid and standard induction chemotherapy for patients aged over 1 year with stage 4 neuroblastoma: A randomised trial. Lancet Oncol 9: 247-256, 2008 .
24. Kreissman SG, Seeger RC, Matthay KK, London WB, Sposto R, Grupp SA, Haas-Kogan DA, Laquaglia MP, Yu AL, Diller L, et al: Purged versus non-purged peripheral blood stem-cell transplantation for high-risk neuroblastoma (COG A3973): A randomised phase 3 trial. Lancet Oncol 14: 999-1008, 2013.

25. Li H, Jin X, Zhang Z, Xing Y and Kong X: Inhibition of autophagy enhances apoptosis induced by the PI3K/AKT/mTor inhibitor NVP-BEZ235 in renal cell carcinoma cells. Cell Biochem Funct 31: 427-433, 2013

26. Echeverry N, Ziltener G, Barbone D, Weder W, Stahel RA, Broaddus VC and Felley-Bosco E: Inhibition of autophagy sensitizes malignant pleural mesothelioma cells to dual PI3K/mTOR inhibitors. Cell Death Dis 6: e1757, 2015.

27. Ji Y, Di W, Yang Q, Lu Z, Cai W and Wu J: Inhibition of autophagy increases proliferation inhibition and apoptosis induced by the PI3K/mTOR inhibitor NVP-BEZ235 in breast cancer cells. Clin Lab 61: 1043-1051, 2015.

28. Jiang X, Overholtzer $M$ and Thompson CB: Autophagy in cellular metabolism and cancer. J Clin Invest 125: 47-54, 2015.

29. Fulda S and Kögel D: Cell death by autophagy: Emerging molecular mechanisms and implications for cancer therapy. Oncogene 34: 5105-5113, 2015.

30. Rodríguez CE, Reidel SI, Bal de Kier Joffé ED, Jasnis MA and Fiszman GL: Autophagy protects from trastuzumab-induced cytotoxicity in HER2 overexpressing breast tumor spheroids. PLoS One 10: e0137920, 2015.

31. Song J, Qu Z, Guo X, Zhao Q, Zhao X, Gao L, Sun K, Shen F, Wu M and Wei L: Hypoxia-induced autophagy contributes to the chemoresistance of hepatocellular carcinoma cells. Autophagy 5: 1131-1144, 2009

32. Toshima T, Shirabe K, Matsumoto Y, Yoshiya S, Ikegami T, Yoshizumi T, Soejima Y, Ikeda T and Maehara Y: Autophagy enhances hepatocellular carcinoma progression by activation of mitochondrial $\beta$-oxidation. J Gastroenterol 49: 907-916, 2014

33. Goussetis DJ, Altman JK, Glaser H, McNeer JL, Tallman MS and Platanias LC: Autophagy is a critical mechanism for the induction of the antileukemic effects of arsenic trioxide. J Biol Chem 285: 29989-29997, 2010.

34. Crazzolara R, Cisterne A, Thien M, Hewson J, Baraz R, Bradstock KF and Bendall LJ: Potentiating effects of RAD001 (Everolimus) on vincristine therapy in childhood acute lymphoblastic leukemia. Blood 113: 3297-3306, 2009. 